\title{
Performance Comparison on Trust Based Power Aware Reliable On Demand Routing Protocol in Mobile Ad Hoc Networks
}

\author{
Mukesh Kumar Garg ${ }^{1}$, Dharam Vir ${ }^{2}$, Dr. S.K. Agarwal ${ }^{3}$ \\ ${ }^{1}$ (Department of Computer Engineering, YMCA University of Science \& Technology, Faridabad,India) \\ 2, 3 (Department of Electronics Engineering, YMCA University of Science \& Technology, Faridabad, India)
}

\begin{abstract}
A mobile ad hoc network (MANET) is a collection of mobile wireless nodes, which communicate to each other without any centralized administration. Trust and Power consumption is the most challenging task in routing protocol design for Mobile ad hoc networks (MANETs), since the highly dynamic nature of MANET coupled with limited bandwidth and battery power imposes severe restrictions on routing protocols especially on achieving the routing. Furthermore replacing and recharging batteries and making nodes co-operative are often impossible in significant environments. In this paper, we effort to study the performance of three ondemand routing protocols (AODV, DSR, DYMO) in terms of number of hop counts, number of routes selected, number of RREQ packets, number of RREP packets and power consumption in transmit, received and ideal modes. During route discovery, nodes are more trust to find reliable route for communication and utilizing maximum energy capacity for selection of route. Route based selection of nodes called 'Reliability'. Route request from the source is accepted by a node only if its reliability is high. Otherwise, the route request is discarded. This approach forms a reliable route from source to destination thus increasing network life time, improving power utilization and decreasing number of packet loss during transmission. To solve the above problem here we describe the three on demand routing and it is simulated using QualNet 5.0 simulator.
\end{abstract}

Keywords: AODV, DSR, DYMO, Mobile Ad Hoc Networks, Power, Reliability, Trust

\section{INTRODUCTION}

Mobile Ad Hoc Networks are formed by devices that are able to communicate with each other using a wireless physical medium without having a route to a pre existing network infrastructure [1] [2]. A fundamental characteristic of ad hoc networks is that they are able to configure themselves on-the-fly without the involvement of a centralized administrator. Although all wireless networks as shown in Fig 1 work without any physical connection but with a fixed infrastructure The increasing use of wireless portable devices such as mobile phones and laptops as part of everyday life, is leading to the possibility for unstructured or ad hoc wireless communication. With these types of devices, there is a fundamental ability to share information. There is no need of access points, each node act as a router and node at the same time. These mobile nodes (router) can leave and join the network according to their own wish. Every node finds the route-by-route request. Routing protocol plays an important role to send the data from source to destination that discovers the optimal path between the two communication nodes [6].

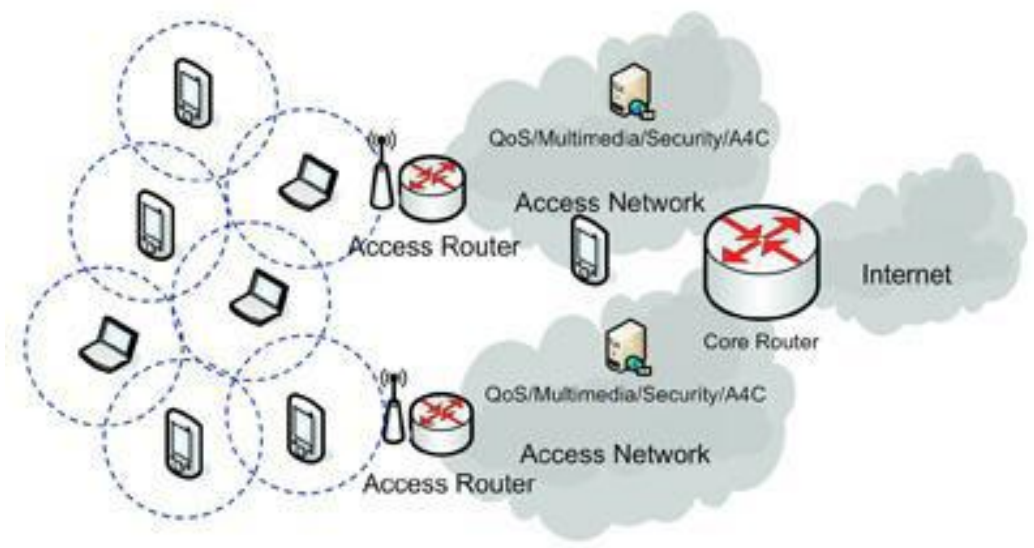

Fig. 1 An example of various wireless networks

Every protocol has its own rules to finds the route or maintenance the route. There are various routing protocol proposed by researchers. Routing protocols in MANETs are generally classified as pro-active and reactive. The re-active protocols had gained more attraction as it reduces routing overheads. Many of the work reported on routing protocols have focused only on shortest path, limited power ability, Mobility models, 
transmission power limit, No central controlling authority, continuously maintains the information required properly route traffic, power aware and minimum cost. However much or less attention has been paid in making the routing protocol to choose a more reliable route. In critical environments like military operations, data packets are forwarded to destination through reliable intermediate nodes. Hence our work focuses on augmenting the existing on-demand routing protocols and making them reliable. We have used AODV (Ad hoc on-demand distance vector) DSR (Dynamic source routing) and DYMO (Dynamic MANET On-demand) protocol as the base on-demand routing protocol for our model. We have used the means of simulation using QualNet 5.0 simulator to gather data about these routing protocols in order to evaluate their performance [1][7].

The rest of this paper is organized as follows: in section II problem formulation and major issues. In section III trust based power aware on demand routing protocols is discussed. In section IV Simulation setup and platform used in this work is discussed. In section $\mathrm{V}$ the results of the performance evaluation are thoroughly discussed. Conclusion and future work is given in section VI.

\section{Problem Formulation AND MaJOR ISSUES}

The key issue with ad-hoc networking is how to send a message from one node to another with no direct link. The nodes in the network are moving around randomly, and it is very difficult that which nodes are directly linked together and the intermediate node judges its ability to forward the RREQ packets or drop it [8]. The number of packets transferred successfully by each node. Route from source to destination is determined by selecting the most trusted path [3]. Here battery capacity is not considered as an issue for selecting the path between source and destination. Same time topology of the network is constantly changing and it is very difficult for routing process. We efforts to simulate and analyze of these two parameters to discover a reliable route between the source and destination and reduce power consumption. A number or routing protocols are available at present; some of them are taken below for discussion purpose [17].

\section{A. ROUTING PROTOCOLS:}

\section{Trust Based Power Aware Aodv, DSR, Dymo Protocols}

Routing protocols in MANETs are classified into three different categories according to their functionality and performance.

1. Proactive (Table driven) protocols

2. Reactive (On-demand) protocols

3. Hybrid protocols

Table Driven Routing Protocols: It is also known as proactive routing protocols. In these protocols, the routing information is stored in the structure of tables maintained by each node. These tables need to be updated due to frequent change in the topology of the network. These protocols are used where the route requests are frequent. Example: FSR, GSR, DSDV, STAR, CGSR, OLSR, WRP [1] [17].

On Demand Routing Protocols: It is also known as reactive protocols. They involve discovering routes to other nodes only when they are needed. A route discovery process is invoked when a node wishes to communicate with another for which it has no route table entry. They are generally considered efficient protocol, where the route discovery is required to be less frequent. This makes them more suitable to the network with light traffic and low mobility. Example: DSR, AODV, LAR, TORA, CBRP, ARA [17].

Hybrid Routing Protocols: These protocols combine the advantages of the two routing protocols in order to obtain higher efficiency. In these protocols network is divided in to the zones, if the routing is to be carried out within the zone than table driven routing is used otherwise on demand routing is preferable. Example: ZRP, DST, DDR, ZHLS [17].

\section{B. ROUTING PROTOCOLS UNDER CONSIDERATION:}

The Ad Hoc routing protocols are mainly classified in three categories discussed in above section and shown in fig 2. This section provides the routing technique for the protocols which we are used in our simulation. This section also presents a novel efficient on demand routing algorithm that generates a reliable route between source and destination [4] [9]. 


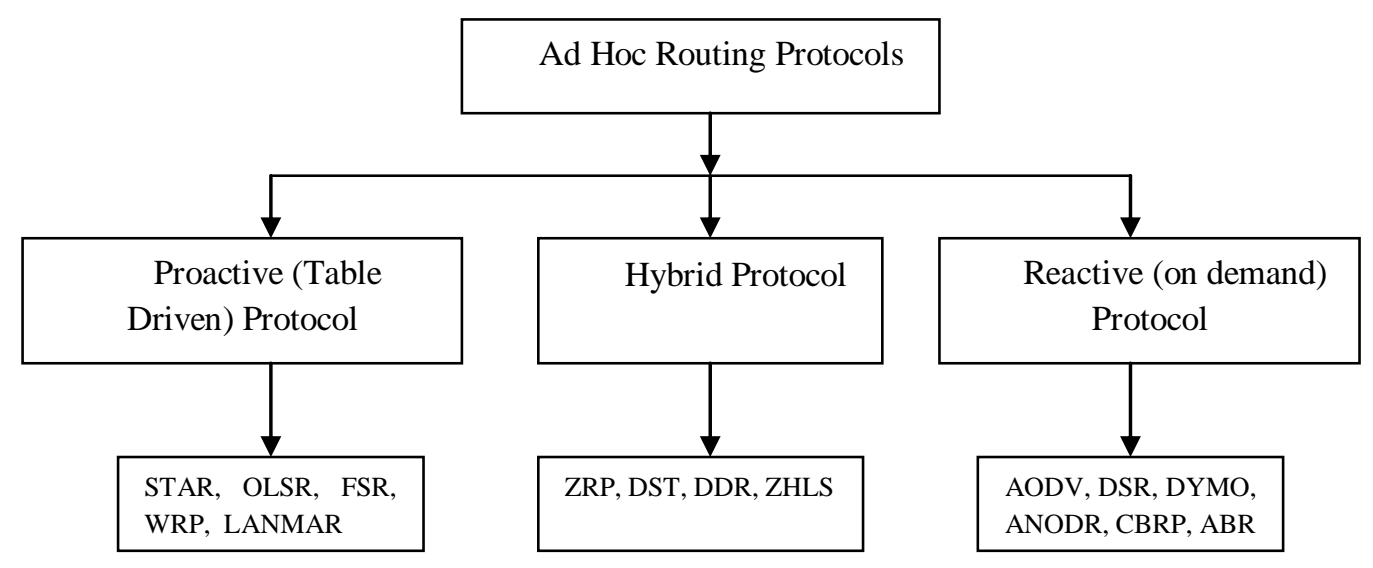

Fig. 2 Classification of Ad-hoc routing protocol

\section{1) Overview of Ad hoc on demand distance vector (AODV):}

It is one of the most popular MANET routing protocols named as re-active or on-demand routing protocol. Ad hoc on demand distance vector (AODV) routing protocol creates routes on-demand. In AODV, a route is created only when requested by a network connection and information about to this route is stored only in the routing tables of those nodes that are present in the path of the route. The process of route establishment is as follows. Assume that node A wants to set up a connection with node B. Node A initiates a path discovery process in an effort to establish a route to node B by broadcasting a Route Request (RREQ) packet to its immediate neighbors. Each RREQ packet is identified through a combination of the transmitting node's IP address and a broadcast through identification [12]. The latter is used to identify different RREQ broadcasts by the same node and is incremented for each RREQ broadcast. Therefore, each RREQ packet carries a sequence number which allows intermediate nodes to reply to route requests only with up-to-date route information. Ahead reception of an RREQ packet by a node, the information is forwarded to the immediate neighbors of the node and the procedure continues until the RREQ is received either by node B or by a node that has recently established a route to node B. If subsequent copies of the same RREQ are received by a node, these are discarded. When a node forwards a RREQ packet to its neighbors, it records in its routing table the address of the neighbor node where the first copy of the RREQ was received. This helps the nodes to establish a reverse path, which will be used to carry the response to the RREQ. AODV supports only the use of symmetric links. A timer starts running when the route is not used. If the timer exceeds the value of the 'lifetime', then the route entry is deleted. Routes may change due to the movement of a node within the path of the route. In such a case, the upstream neighbor of this node generates a 'link failure notification message' which notifies about the deletion of the part of the route and forwards this to its upstream neighbor. The procedure continues until the source node is notified about the deletion of the route part caused by the movement of the node. Upon reception of the 'link failure notification message' the source node can initiate discovery of a route to the destination node [1] [12].

\section{2) Overview of Dynamic Source Routing (DSR):}

Dynamic Source Routing (DSR) [13] uses source routing rather than hop-by-hop routing therefore; we designed a scenario for DSR multi-hop wireless networks with varying number of nodes. DSR contains two mechanisms of route discovery and route maintenance. The route discovery phase initiate when source does not know route to the destination. When source sends a message to destination it first search it into the route cache if not found it generates a RREQ message and work in RREQ/RREP mode. Route cache is also maintained for the purpose of storing old routes. DSR every packet to be routed carries in its header the ordered list of network nodes that constitute the route over which the packet is to be relayed. Thus, intermediate nodes do not need to maintain routing information as the contents of the packet itself are sufficient to route the packet. This fact eliminates the need for the periodic route advertisement and neighbor detection packets that are employed in other protocols. DSR supports both symmetric and asymmetric links. Thus, the RREP message can be either accepted over the same path with original RREQ, or the destination node might initiate its own route discovery towards the source node and piggyback the RREP message in its RREQ. In order to limit the overhead of these control messages, each node maintains a cache comprising routes that were either used by these nodes or overheard. As a result of route request by a certain node, all the possible routes that are learned are stored in the cache. Thus, a RREQ process may result in a number of routes being stored in the source node's cache. Route maintenance is initiated by the source node upon detection of a change in network topology that prevents its packet from 
reaching the destination node. Therefore route recovery in DSR can be faster than any other on-demand routing protocols. Since route maintenance is initiated only upon link failure, DSR does not make use of periodic transmissions of routing information, resulting in less control signaling overhead and less power consumption at the mobile nodes [1][6].

\section{3) Overview of Dynamic Manet on-Demand (DYMO):}

The Dynamic MANET On-Demand (DYMO) protocol is a simple and fast routing protocol for multihop networks. It discovers unicast routes among DYMO routers within the network in an on-demand approach, offering improved convergence in dynamic topologies. To ensure the precision of this protocol, digital signatures and mix up chains are used. The basic operations of the DYMO protocol are route discovery and route management. The following sections explain these mechanisms in more details [17].

a)

\section{Route discovery process:}

When a source needs to send a data packet, it sends an RREQ to discover a route to that particular destination shown in fig. 3. After issuing an RREQ, the origin DYMO [14] router waits for a route to be discovered. If a route is not obtained within RREQ waiting it may again trying to discover a route by issuing another RREQ. This buffer should have a fixed limited size and older data packets should be discarded first. Buffering of data packets can have both positive and negative effects, and therefore buffer settings should be managerially configurable or intelligently controlled. If a route discovery has been attempted maximum times without receiving a route to the target node, all data packets proposed for the corresponding target node are dropped from the buffer and a destination inaccessible ICMP message is delivered to the source [11].

\section{b) Route maintenance:}

When a data packet is to be forwarded and it can not be delivered to the next-hop because no forwarding route for the IP destination address exists, an RERR is issued is based on condition, an ICMP destination unreachable message must not be generated except this router is responsible for the IP destination address and that IP destination address is known to be unreachable. Moreover, an RERR should be issued after detecting a broken link of a forwarding route and quickly notify DYMO routers that a link break occurred and that certain routes are no longer available. If the route with the broken link has not been used recently, the RERR should not be generated [14].

\section{Simulation SeTUP AND Models}

We have used a simulation model based on QualNet 5.0 Simulator [15], with Graphical User Interface tools for performance analysis comparison. QualNet Developer is ultra high-fidelity network evaluation software that predicts wireless, wired and mixed-platform network and networking device performance. QualNet runs on sequential and parallel UNIX, Windows, Mac OS X and Linux operating systems, and is also designed to link with modeling/simulation applications and live networks. The simulator contains standard API for composition of protocols across different layers. QualNet support a wider range of networks and their analysis, some of them are MANET, QoS, Wired Networks, Satellite and cellular [16]. The simulation parameters for design a scenario which have been considered for the analysis of on demand routing protocols (AODV, DSR, and DYMO) are given below in Table 1. A scenario is designed for trust based power aware routing protocol using all three protocols, after running the scenario program snapshot is obtained shown in fig. 3.

Table 1: Power and Mobility traffic model parameters for AODV, DSR and DYMO routing protocol.

\begin{tabular}{|l|l|}
\hline \multicolumn{1}{|c|}{ Parameter } & \multicolumn{1}{c|}{ QUalue } \\
\hline Simulator & AODV, DSR, DYMO \\
\hline Routing Protocols & IEEE 802.11 \\
\hline Mac Type & 100 \\
\hline Number of Nodes & $600 \mathrm{~m}$ \\
\hline Transmission range & $30 \mathrm{~s}$ \\
\hline Simulation Time & 1500 X 1500 \\
\hline Simulation Area & Random Waypoint Mobility \\
\hline Mobility Model & \\
\hline
\end{tabular}




\begin{tabular}{|l|l|}
\hline \multicolumn{1}{|c|}{ Parameter } & \multicolumn{1}{c|}{ Value } \\
\hline Traffic Type & Constant-Bit Rate \\
\hline Node Placement Model & Random \\
\hline Battery Model & Linear Model \\
\hline Full Battery Capacity & 1200 (mA,h) \\
\hline Battery Charge Monitoring Interval & 30 Sec. \\
\hline Antenna Models & Omni direction \\
\hline Total packet sent & 24 \\
\hline Packet Size & 12288 Bytes \\
\hline Throughput & 4274 \\
\hline Channel Frequency & 2.4 GHz \\
\hline $\begin{array}{l}\text { Performance Matrices in Physical } \\
\text { Layer }\end{array}$ & $\begin{array}{l}\text { Energy consumed (in mjules) in transmit mode } \\
\text { Energy consumed (in mjules) in received mode }\end{array}$ \\
\hline Energy Model & Mica motes \\
\hline $\begin{array}{l}\text { Energy Supply Voltage } \\
\text { Consumption }\end{array}$ & 6.5 Volt \\
\hline
\end{tabular}

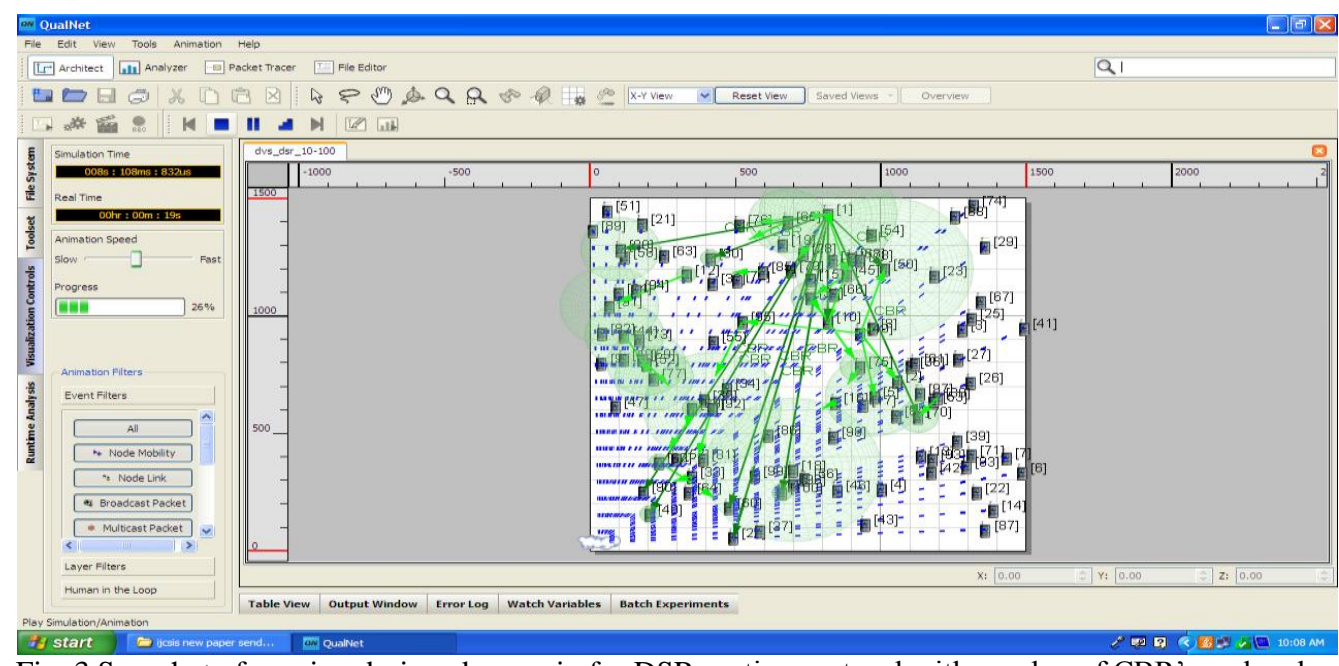

Fig. 3 Snapshot of running designed scenario for DSR routing protocol with number of CBR's and nodes.

\section{A. PERFORMANCE METRICS:}

We have primarily selected the following performance metrics in order to study the reliability on trust based power aware on demand routing protocol in Mobile Ad Hoc networks, performing comparison of AODV, DSR, and DYMO [14][11][10] [5].

- Number of route request packets (RREQ)

- Average time taken to respond to a Route Selection

- Number of Hop count

- Number of route reply packets (RREP)

- Power Consumption in Transmit Mode

- Power Consumption in Received Mode

- Power Consumption in Ideal Mode

- Number of packets forwarded successfully 


\section{RESUlt AND ANALYSIS}

1) Number of reliable route request packets (RRRPQ):

This is defined as the number of routes offered by a routing protocol for an upcoming request.

Fig 4 gives the comparison between reliable routes request packets (RRREQ) selected by on demand routing protocols. Considering the various configured parameters using in table 1 . It has been observed that the AODV routing protocol uses on demand approach for finding routes. The major difference between AODV and DSR stems out from the fact that DSR uses source routing in which a data packet carries the complete path to be traversed, while in AODV the source node and the intermediate node stores the next hop information corresponding to each flow data packet transmission, DYMO trying to routes request and maintained their routes as shown through graphical representation in fig.4.

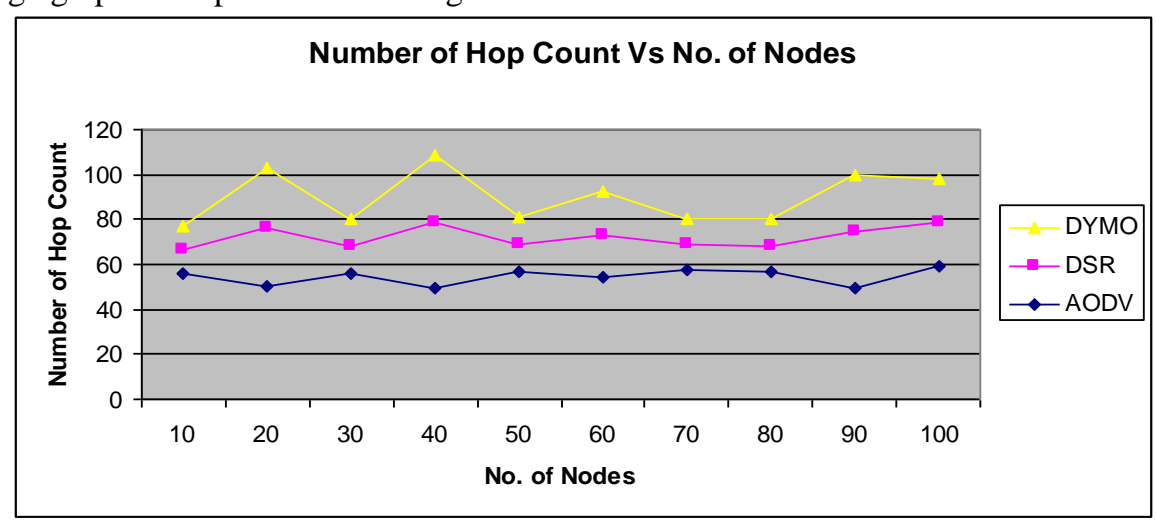

Fig. 4 Comparison of reliable route request packets with varying nodes at different routing protocols.

2) Number of Hop Count:

This is defined as the number of intermediate nodes between a source and destination.

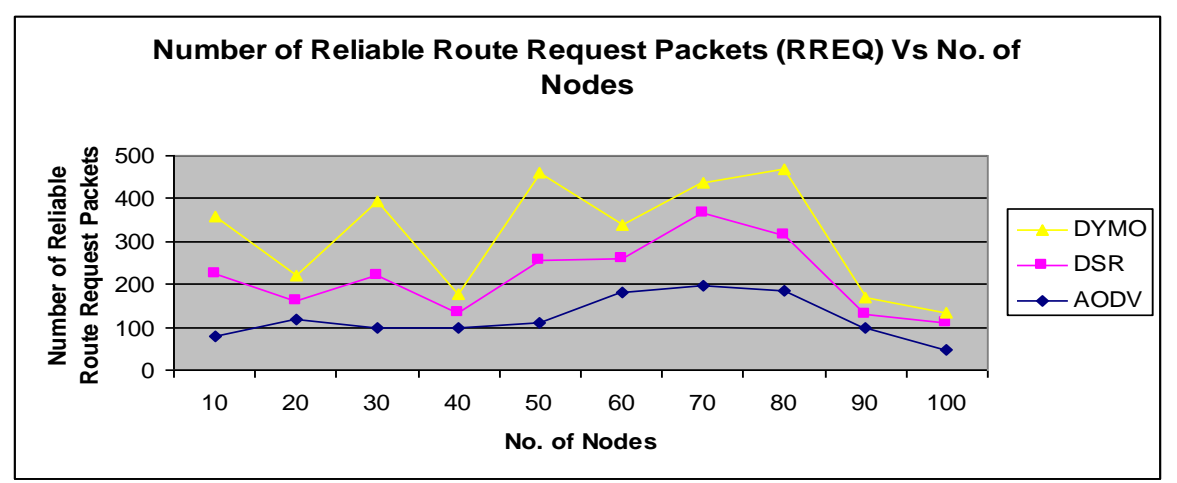

Fig 5. Comparison of Hop counts given by AODV, DSR and DYMO on demand routing protocol

We see that AODV has less number of routes as compared to DSR, which helps AODV to be more efficient and less bulky. Here again we see that AODV has less number of intermediate (nodes between source and destination) nodes in comparison to DSR and DYMO which shows its efficient behavior as we know that more are the intermediate nodes more is the chance of path break and insecure network along with high energy consumption per message transfer by a node.

3) Signal Received and Forward to MAC:

Number of signals received by the physical layer process and subsequently forwarded to the MAC layer for further processing. 


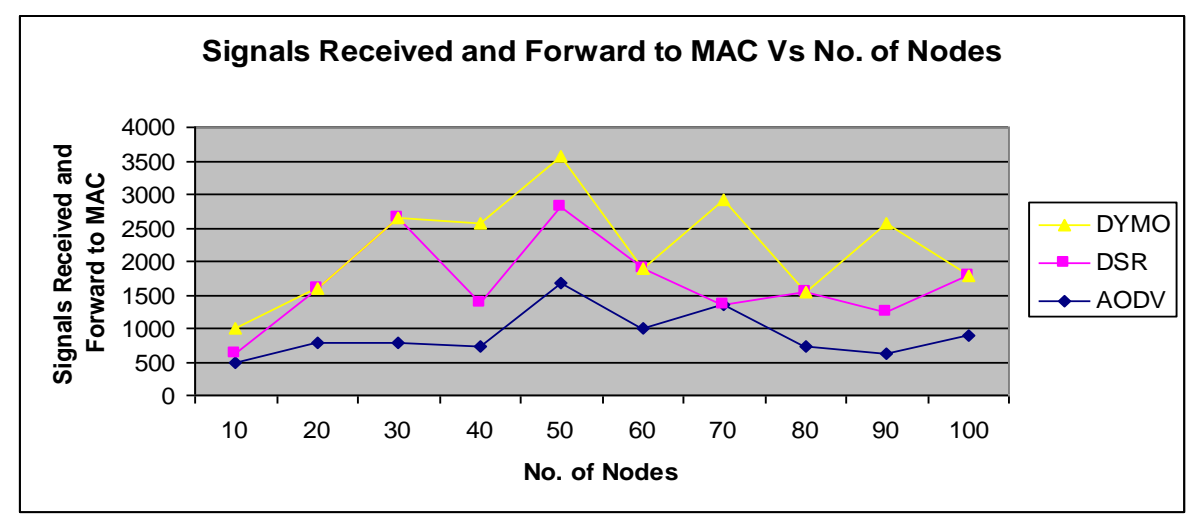

Fig 6. Comparison of Signal Received and Forward to MAC given by AODV, DSR and DYMO with varying no of nodes on demand routing protocol

From fig. 6 here again we see that AODV has less number of packets and immediately forward to source and destination nodes in comparison to DSR and AODV shows its efficient behavior as we know that more are the intermediate nodes more is the chance of path break and also increases the efficiency of networks.

4) Number of route reply packets (RREP):

Total number of route replies received by a node.

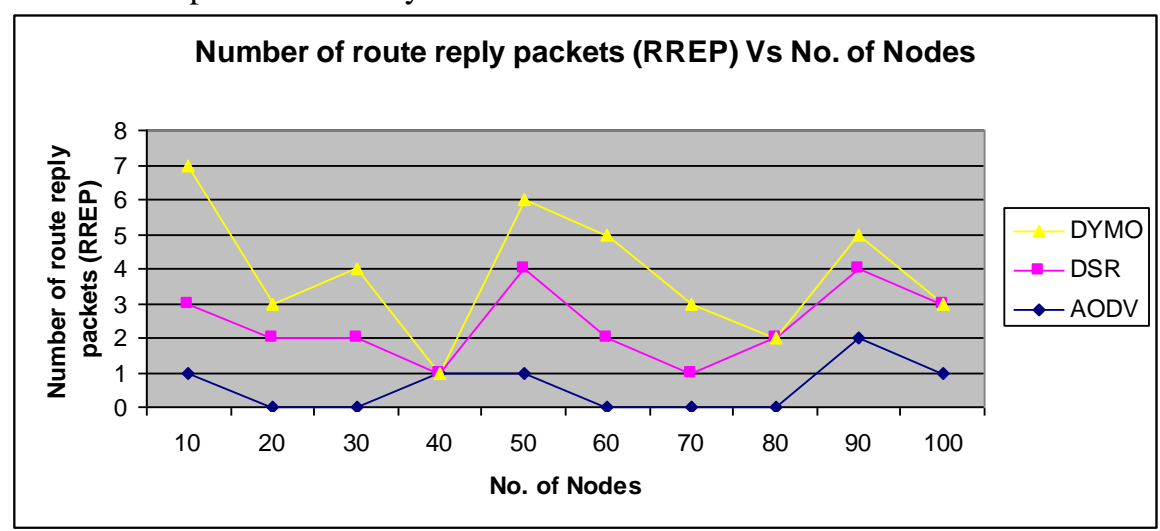

Fig 7. Comparison of number of route reply packets of AODV, DSR and DYMO with varying no of nodes on demand routing protocol.

Fig 7 is the comparison of route reply packets made by AODV and DSR here we see that AODV has more route reply options as compared to DSR; also DSR maintains multiple routes to the same destination in the cache. But unlike AODV, DSR and DYMO has no mechanism to determine the freshness of the routes. It also does not have any mechanism to expire the stale routes. With high mobility, link breaks are frequent and there is the possibility of more routes becoming stale quickly. This requires the DSR to initiate the route discovery process which further adds to the increasing delay. From here also we can see that AODV is more efficient as compared to DSR and DYMO.

\section{B. Power Consumption:}

This is the ratio of the average power consumed in each node to total power. The lifetime, scalability, response time and effective sampling frequency, all of these parameters of the wireless sensor network depend upon the power. Power failure regularly breaks in the network. Energy is required for maintaining the individual health of the nodes, during source to destination receiving the packets and transmitting the data as well. 
1) Power consumed in reliable transmit mode:

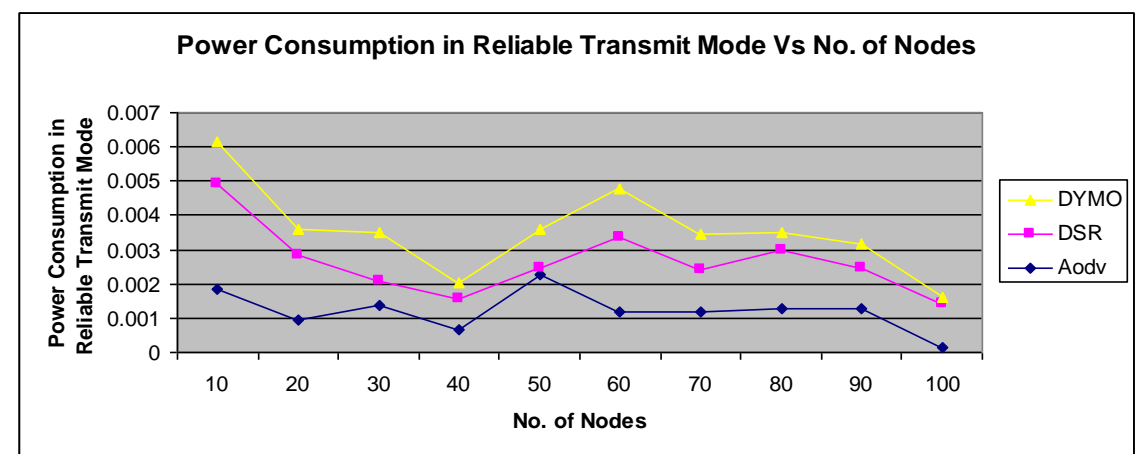

Fig. 8 Power consumed in reliable transmit mode with varying nodes different routing protocols.

- Fig. 8 shows the impact variation of Power consumption in reliable transmit mode with varying number of nodes (10 to 100) in equal. Following inference can be made:

- By observation from graph and running scenario the reliable maximum power consumed when distance of nodes is longer, hence overall performance of power manage by AODV tailed by DSR and DYMO.

- $\quad$ The maximum power consumes by DYMO.

2) Power consumed in reliable received mode:

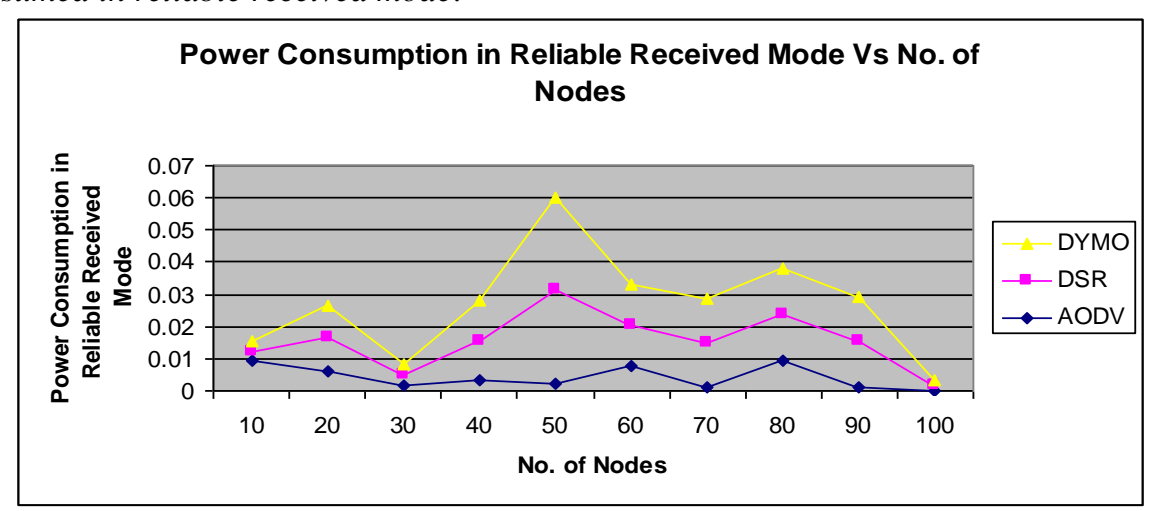

Fig. 9 Power consumed in reliable received mode with varying nodes different routing protocols.

- Fig. 9 shows the impact variation of reliable power consumption in received mode with variation in number of nodes. Following inference can be made:

- On analyzing the results for energy consumption in reliable receive mode it has been concluded that AODV consumes less power as compare to DYMO and DSR.

- Maximum average power consumes when long distance nodes communicate each other as observing graph and Scenario in figure 3.

3) Power consumption in reliable ideal mode:

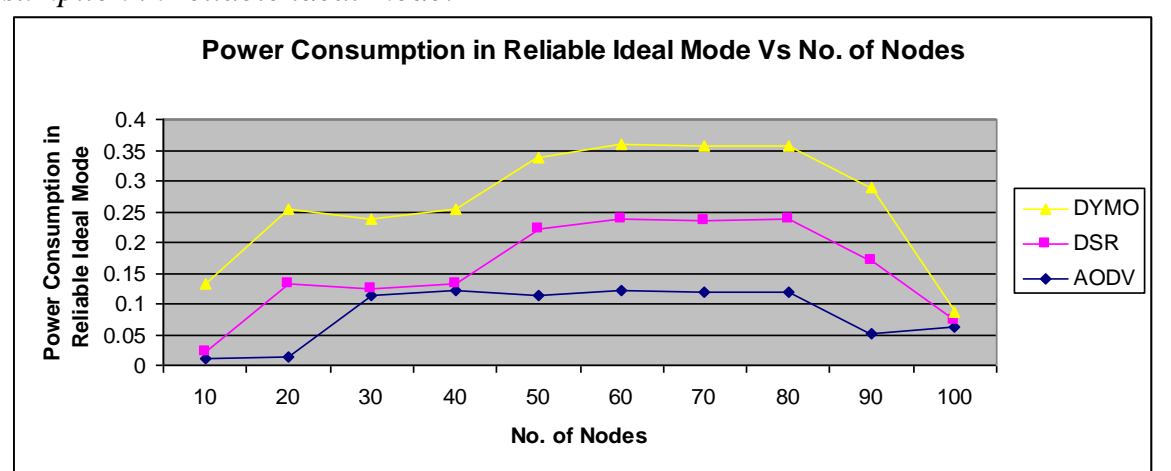

Fig. 9 Power consumed in reliable ideal mode with varying nodes different routing protocols.

- Fig. 9 shows the impact variation of reliable power consumption in ideal mode with variation number of nodes. Following inference can be made: 
- By observation from graph and running scenario the over all power consumed when distance of nodes is increases and takes long distance between source and destination.

- Hence overall performance of power manage by ADDV is good enough tailed by DYMO than DSR.

- The maximum power consumes by DSR which is tailed by manage AODV and DYMO.

\section{CONCLUSION AND FUTURE WORK}

Simulation on QualNet shows that AODV compared with DSR and DYMO reduces the number of hop count nodes, and AODV has less number of routes as compared to DSR, which helps AODV to be more efficient and less bulky. While comparing route request packets AODV is again better by having more number of RREQ packets as compared to DSR and DYMO which made it more efficient in finding a new route and each time in replacing a stale link propose a trust based power aware routing model in MANET. AODV protocol can work in critical environment like military scenarios because of decreases packet drops and improves reliability . The life time of the network and each node is increased by choosing more reliable node as a router to route the packets. Our future work will highlight the mobility issues on reliability and power management in hybrid routing protocols.

\section{ACKNOWLEDGMENT}

The authors wish to acknowledge Varinder Singh, YMCA University of Science \& Technology, Faridabad, India, for his continual support to carry out this work.

\section{REFERENCES}

[1] LIU Jian and LI Fang-min, "An Improvement of AODV Protocol Based on Reliable Delivery in Mobile Ad hoc Networks", Fifth International Conference on Information Assurance and Security, 2009.

[2] Rekha Patil , A.Damodaram, "Cost Based Power Aware Cross Layer Routing Protocol For Manet", IJCSNS International Journal of Computer Science and Network Security, VOL.8 No.12, December2008.

[3] M.Tamilarasi, T.G Palani Velu, "Integrated Energy-Aware Mechanism for MANETs using On-demand Routing", International Journal of Computer, Information, and Systems Science, and Engineering 2; www. waset.org Summer 2008.

[4] M.Pushpalatha, Revathi Venkatraman, "Security in Ad Hoc Networks: An extension of Dynamic Source Routing", 10th IEEE Singapore International conference on Communication Systems Oct 2006,ISBN No:1-4244-0411-8,Pg1-5.

[5] V. Rishiwal, M. Yadav and S. Verma, "Power Aware Routing to support Real Time Traffic in Mobile Adhoc Networks", International Conference on Emerging Trends in Engg \& Tech., IEEE, pp. 223-227, 2008

[6] Huafeng Wu1, Chaojian Shi1," A Trust Management Model for P2P File Sharing System", International Conference on Multimedia and Ubiquitous Engineering, IEEE Explore 978-0-7695-3134-2/08, 2008.

[7] LIXin MIAO Jian song," A new traffic allocation algorithm in AD hoc networks, "The Journal of China University of Post and Telecommunication", Volume 13. Issue 3. September 2006.

[8] P. Sinha, R. Sivakumar, and V. Bharghavan(1999), CEDAR: A Core Extraction Distributed Ad-hoc Routing Algorithm. IEEE Journal on Selected Areas in Communications, vol. 17, no. 8, pp. 1454-1466.

[9] Smt Rajashree.V. Biradar \& Prof V. C.Patil : "Classification and Comparison of routing Techniques in Wireless Ad-hoc Networks", in the proceedings of international Symposium on Adhoc Ubiquitous Computing (ISHUC'06), pp. 7-11 (2006).

[10] Robert Castaneda and Samir R. Das(1999): “Query Localization Techniques for On-demand Routing Protocols in Ad-hoc Networks" ,. In Proceedings of the Fifth International Conference on Mobile Computing and Networking (MobiCom'99). ACM.

[11] C.F. Chiasserini, I. Chlantac, P.Monti, and A.Nucci, "Energy - Efficient Design of Wireless Ad Hoc Networks", Proceedings of Networking 2002, pp. 376-386, May 2002.

[12] Perkin, C.E and E.M Royer, “Ad hoc on Demand Distance Vector Routing”, Proceeding of 2nd IEEE Workshop, Mobile Computing, Sys. Applications, pp: 90-100, 1999.

[13] SreeRangaRaju, JitendranathMungara, "ZRP Versus AODV and DSR: A Comprehensive Study on ZRP Performance Using Qualnet Simulator”, IEEE Conference WiCOM 2010.

[14] Sumit Kumar Singh, Shiva Prakash and Kapil Kumar, "Energy Aware Dynamic MANET On-demand (EA-DYMO)", International Journal of Computer Applications, July 2011.

[15] QualNet documentation, "QualNet 5.0 Model Library:Wireless"; http://www.scalablenetworks.com,

[16] “Qualnet 5.0,” http://www.scalable-networks.com,

[17] Wireless routing protocols www.wikipedia.com 